NASA Technical Memorandum 107075

ICOMP-95-18

19960007783

CEAS/AIAA-95-144

\title{
A Zonal Approach for Prediction of Jet Noise
}

S.H. Shih and D.R. Hixon

Institute for Computational Mechanics in Propulsion

Lewis Research Center

Cleveland, Ohio

Reda R. Mankbadi

Lewis Research Center

Cleveland, Ohio

Prepared for the

First Joint Aeroacoustics Conference

cosponsored by the American Institute of Aeronautics and Astronautics and the German Society for Aeronautics and Astronautics

Munich, Germany, June 12-15, 1995

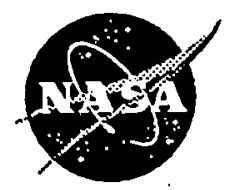

National Aeronautics and Space Administration

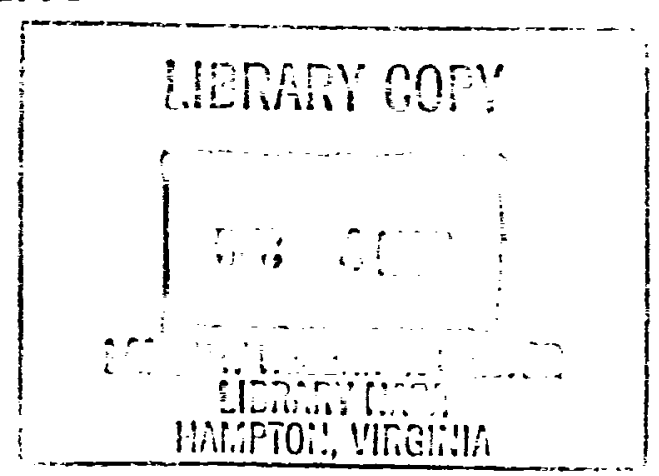




\title{
A Zonal Approach for Prediction of Jet Noise
}

\author{
S. H. Shih* and D. R. Hixon* \\ Institute for Computational Mechanics in Propulsion \\ NASA Lewis Research Center \\ Cleveland, Ohio 44135 \\ R. R. Mankbadi** \\ NASA Lewis Research Center \\ Cleveland, Ohio 44135
}

\begin{abstract}
A zonal approach for direct computation of sound generation and propagation from a supersonic jet is investigated. The present work splits the computational domain into a non-linear, acoustic-source regime and a linear acoustic wave propagation regime. In the non-linear regime, the unsteady flow is governed by the largescale equations, which are the filtered compressible Navier-Stokes equations. In the linear acoustic regime, the sound wave propagation is described by the linearized Euler equations. Computational results are presented for a supersonic jet at $M=2.1$. It is demonstrated that no spurious modes are generated in the matching region and the computational expense is reduced substantially as opposed to fully large-scale simulation.
\end{abstract}

\section{Introduction}

In theory, direct numerical simulation (DNS) based on the compressible Navier-Stokes equations provide both the flow fluctuations and the acoustic field. Howev$e r$, the resolution requirement for high-Reynolds number turbulent flows makes direct numerical simulation impractical due to current computer limitations. It is known that the large scale structure is responsible for the generation of the dominant part of supersonic jet noise [1-6]. This indicates that it is appropriate to perform large-eddy simulations(LES) to accurately capture the large scales of motion while modelling the sub-grid scale turbulence.

The use of large-eddy simulations (LES) as a tool for prediction of the jet noise source has been proposed

\footnotetext{
* Senior Research Associate, member AIAA

** Senior scientist and technical leader, CAA, Associate fellow AIAA

This paper is declared a work of the U.S. Government and is not subject to copyright protection in the United States
}

by Mankbadi et al. [7], in which, the computed fluctuating sound source in the near field was then used to obtain the far field sound through the application of Lighthill's theory. Due to the non-compactness of the source and the need to account for the retarded time, accurate application of Lighthill's theory requires prohibitive computer storage. Later, Mankbadi et al. [8] extended the computational domain to the acoustic field, where large-scale equations are solved for both the sound source and the acoustic fields for the axisymmetric case. The approach was extended in Shih et al. [9] to the three dimensional case, but was restricted to the near region due to computer limitations. An altemative approach for extending the three dimensional computation to the far-field is needed. This issue is addressed in this paper.

An acoustic calculation can be viewed as consisting of two parts, one describing the non-linear generation of sound, the other describing the linear propagation of sound. All non-linear flow effects and source generation are confined to the near field, and can be computed by large-scale equations. There are several approaches to calculate the sound propagation once the source has been identified, such as acoustic analogy, Kirchhoff's method [10], and linearized Euler equations. Freund et al. [11] have studied the matching of near/far-field equation sets for computations of aerodynamic sound. Their results of an acoustic source embedded in a shear layer using linearized Euler equations as the far-field equation set are encouraging.

In the present work, the near-field source region solution including all non-linear flow hydrodynamics is obtained through the large-scale equations [8], and is matched to the solution of the linearized Euler equations governing the acoustic field. The present research combines the large-scale simulation and linearized Euler equations approach into one computer code, resulting in the saving of computer CPU time for extension to three dimensional acoustic field predictions. 


\section{Governing Equations}

The computational domain, figure 1 , is split into non-linear source generation and linear acoustic propagation regions, which are governed by the large-scale and linearized Euler equations respectively.

\subsection{Large-Scale Equations}

The flow field of a supersonic jet is governed by the compressible Navier-Stokes equations, and can be decomposed into filtered and residual fields, namely

$$
\mathbf{f}=\mathbf{f}+\mathbf{f}^{\mathbf{*}}
$$

where an overbar denotes the resolved (filtered) field and a (") denotes the umresolved (subgrid) one. The mean of the filtered field is the mean of the total field. Upon substimting this splitting in the full Navier-Stokes equations, the filtered compressible Navier-Stokes equations in cylindrical coordinates takes the form

$$
\frac{\partial Q}{\partial t}+\frac{\partial F}{\partial x}+\frac{1}{r} \frac{\partial}{\partial r}(r G)=S
$$

where

$$
Q=\left[\begin{array}{llll}
\bar{\rho}, & \bar{\rho} \bar{u}, & \rho \bar{v}, & \bar{\rho} \hat{E}
\end{array}\right]^{T}
$$

$F=\left[\begin{array}{c}\bar{\rho} \bar{u} \\ \rho+\bar{\rho} \bar{u}^{2}-\bar{\sigma}_{x x}-\tau \\ \rho \bar{u} \bar{v}-\tilde{\sigma}_{x x}-\tau x r \\ \tilde{\rho} \bar{u} \tilde{I}-\bar{u} \bar{\sigma}_{x x}-\bar{v} \bar{\sigma}_{x r}-k \frac{\partial}{\partial x} \bar{T}-c_{v} q\end{array}\right]$

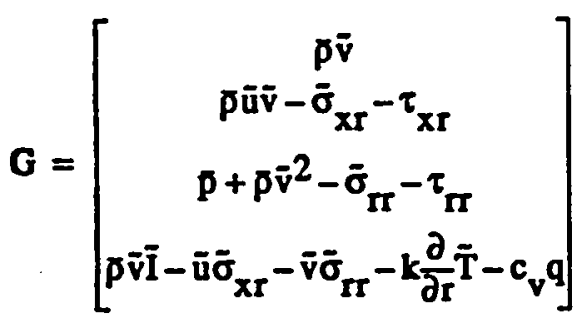

$$
S=\frac{1}{x}\left[\begin{array}{c}
0 \\
0 \\
p-\tilde{\sigma}_{\phi \phi}-\tau_{\phi \phi} \\
0
\end{array}\right]
$$

Here $Q$ is the unknown vector, $F$ and $G$ are the fluxes in the $x$ and $r$ directions, respectively; $S$ is the source term that arises in cylindrical polar coordinates; and $k$ is thermal conductivity. The total enthalpy is $I$, the total energy is $E$, and $\sigma_{i j}$ are the viscous stresses. This system of equations is coupled with the equation of state for a perfect gas. Here, a tilde denotes Favre fitering,

$$
\overline{\mathrm{f}}=\frac{\overline{\mathrm{ef}}}{\overline{\mathrm{p}}}
$$

The unresolved stresses $\tau_{\mathrm{ij}}$ appearing in equations (4-6) need to be modeled.

\section{2,2 Linearized Euler Equations}

Starting from the full Navier-Stokes equations in conservation form, neglecting viscosity, and linearizing about a mean flow (U,V), the axisymmetric linearized Euler equations may be written in cylindrical coordinates as:

$$
\frac{\partial Q}{\partial t}+\frac{\partial F}{\partial x}+\frac{1}{r} \frac{\partial}{\partial r}(r G)=S
$$

where

$Q=[\hat{\rho}, \hat{u}, \hat{v}, \hat{e}]=\left[\rho^{\prime},(\rho u)^{\prime},(\rho v)^{\prime},(\rho e)^{\prime}\right]$

$F=\left[\begin{array}{c}\hat{u} \\ \hat{p}^{\prime}+2 \hat{u} U-\hat{\rho} U^{2} \\ \hat{u} V+\hat{v} U-\hat{\rho} U V \\ \left(p^{\prime}+\hat{e}\right) U+(\hat{u}-\hat{\rho} U) E\end{array}\right]$

and

$$
\mathrm{p}^{\prime}=(\gamma-1)\left[\hat{\mathrm{e}}-(\hat{\mathrm{u} U}+\hat{\mathrm{v} V})+\frac{1}{2} \hat{\rho}\left(\mathrm{U}^{2}+\mathrm{v}^{2}\right)\right]
$$

In this notation, $U$ and $V$ are the mean axial and radial velocities. The velocities are normalized by the jet exit centerline velocity $U_{e}$, time by $R / U_{e}$, density by the mean exit centerline value, and pressure by the exit dynamic pressure.

\section{Subgrid-Scale Modelling}

The effect of unresolved scales on the resolved ones is accounted for through the use of Smagorinsky's subgrid-scale model [12]. The subgrid-scale turbulence stresses are represented as follows]: 


$$
\tau_{i j}=-k_{g}\left(\delta_{i j} / 3\right)+2 p v_{R}\left(\tilde{s}_{i j}-\frac{1}{3} \delta_{i j} \tilde{s}_{m m}\right)
$$

where $k_{g}$ is the kinetic energy of the residual turbulence and is neglected with respect to the thermodynamic pressure. The strain rate of the resolved scale is given by

$$
\tilde{S}_{i j}=\frac{1}{2}\left(\frac{\partial \tilde{u}_{i}}{\partial x_{j}}+\frac{\partial \bar{u}_{j}}{\partial x_{i}}\right)
$$

The summation Smm is zero for incompressible flow, $v_{R}$ is the effective viscosity of the residual field,

$$
v_{R}=\left(c_{s} \Delta_{f}\right)^{2} \cdot \sqrt{2 s_{m n} s_{m n}}
$$

and $\Delta_{f}$ is the filter width given by

$$
\Delta_{\mathrm{f}}=\left(\Delta_{\mathrm{X}} \Delta_{\mathrm{r}}\right)^{1 / 2}
$$

For the heat equation, Edison [13] proposed the eddy viscosity model

$$
q=\bar{\rho} \frac{v_{R}}{\operatorname{Pr}_{t}} \frac{\partial \bar{T}}{\partial x_{k}}
$$

where $\mathrm{Pr}_{l}$ is the subgrid-scale turbulent Prandtl number, which can be taken as 0.5 . Smagorinsky's constant $C_{z}$ in equation (16) is 0.1 as was used in previous study [8].

\section{Numerical Method}

The importance of the dispersion and dissipation of a given scheme, used in connection with the computational aeroacoustics, was highlighted by Hardin [14]. Both effects are crucial in computational aeroacoustics, and can render the computed unsteady part of the solution completely unacceptable. As such, high-order accurate schemes are required for problems in computational aeroacoustics.

A fourth-order accurate in space, second-order accurate in time scheme is used, which is an extension of the McCormack scheme by Gottlieb and Turkel [15]. This scheme has been used extensively by other researchers [16-21], to name a few. In this scheme, the operator is split into two one-dimensional operators and applied in a symmetric way to avoid biasing of the solution.

\section{Boundary Conditions}

Boundary condition is an important issue in the computation of jet noise. Proper boundary treatment should allow waves to pass through the boundary without generating reflecting waves. Several boundary treatments were considered [22], and it was shown that the boundary treatments used in [8] were stable, non-reflect- ing, and most suitable for the jet computations. The present work employs the same boundary treatments as in references [8]. The schematic diagram of figure 1 shows various boundary conditions used at each boundary.

In the non-linear acoustic source region, the inflow boundary is split into hydrodynamic disturbance and radiation regimes. A small disturbance, assumed to be mainly hydrodynamic in nature, is introduced at the inflow and is specified from the centerline to $r / D=2$. The disturbance is assumed in the form:

$$
\left[u^{\prime} v^{\prime} p^{\prime} \rho^{\prime}\right]=\Re\left\{\Phi(r) e^{i(\alpha x-\omega t)}\right\}
$$

Orr-Sommerfeld equation are solved to obtain the complex wave number $\alpha$ as the eigenvalue corresponding to the frequency $\omega$ and the radial functions $\Phi(r)$ as the corresponding eigenfunctions.

$$
\Phi(r)=[d(r), \vec{v}(r), \hat{p}(r), \hat{p}(r)]
$$

To obtain the disturbance solution a mean flow must be specified. In the present work, the analytical functions proposed by Tam and Burton [23] to fit the experimental data of Troutt and McLaughlin [24] were used. The mean axial velocity is given by

$\mathrm{U}=1 \quad$ for $\mathrm{r}<\mathrm{h}$

$U=\exp \left[-\ln (2)\left(\frac{r-h(x)}{b(x))}\right)^{2}\right]$ for $D h$

where $b(x)$ is the half-width of the annular mixing layer, and is fitted to the experimental data. The radius of the potential core, $h(x)$, is related to $b(x)$ through the conservation of momentum [23].

For the supersonic regime, all characteristics travel in the flow direction. Thus the primitive variables are given at $x=0$ as outlined above. In the subsonic regime, the following three characteristics are specified according to the linear stability solution:

$$
\begin{aligned}
& p_{t}+\rho c u_{t}=c_{1} \\
& p_{t}-c^{2} \rho=C_{2} \\
& \rho c v_{t}=c_{3}
\end{aligned}
$$

The fourth characteristic is outgoing and is obtained from the interior solution:

$$
p_{t}-\rho c u_{t}=C_{4}
$$

The four characteristic equations are then solved together to obtain the time derivatives of the variables, which are used to update the solution at the inflow boundary.

In the radiation regime $(r / D>2)$, the conventional 
acoustic radiation condition applies:

$$
Q_{t}=-\Gamma(\theta)\left[\frac{x}{R} Q_{x}+\frac{r}{R} Q_{r}+\frac{Q}{R}\right]
$$

where:

$$
\mathbf{Q}=[u, \mathbf{v}, \mathbf{p}, \mathbf{p}]
$$

$R=\sqrt{x^{2}+r^{2}}$

$\Gamma(\theta)=c\left[\frac{x}{R} M+\sqrt{1-\left(\frac{r}{R} M\right)^{2}}\right]$

and $M$ is the local Mach number, $c$ is the sonic velocity. The spatial derivatives which appear in equation (24) are evaluated in an identical manner as the inner flow derivatives.

The outflow treatment is based on the asymptotic analysis of the linearized equations as given by Tam and Webb [25]. The pressure condition is the same as that obtained by Bayliss and Turkel [26], Enquist and Majda [27], and Hariharan and Hagstrom [28], namely:

$$
p_{t}=-\Gamma(\theta)\left[\frac{x}{R} p_{x}+\frac{r}{R} p_{r}+\frac{p}{R}\right]
$$

However, for updating the rest of the primitive variables, Tam and Webb have shown that the momentum and continuity equations should be used to account for the presence of entropy and vorticity waves at the outflow boundary.

For the outflow regime of large radius with the local Mach number less than 0.01 , the outflow condition is replaced by the conventional acoustic radiation condition, equation (24).

The results presented herein are for axisymmetric disturbance, for which the boundary condition at $r=0$ can be stated as

$$
\frac{\partial}{\partial r}[u, p, p]=0 ; v=0
$$

The centerline treatment for non-axisymmetric disturbances is not obvious, and was addressed by Shih et al [9].

In the linear acoustic region, radiation boundary condition of equation (24) is used since only acoustic wave is of significance in this region.

\section{Matching}

In the present work, this scheme is applied to both the large-scale equations and linearized Euler equations. A schematic diagram of the computational domain is presented in Figure 1. No boundary condition is needed in the matching region. The scheme requires fluxes at two grid points outside of the domain to update variables at the boundary. An overlapping region, consisting of 4 grid points in the radial direction, is made to allow variables at interior points to be passed between two domains. The variables of the two solution domains are the filtered quantities and the fluctuation quantities. The mean of the filtered field is the mean of the total field. Therefore, it is straightforward to set up a matching scheme at the interface. At every time step, all linear acoustic region variables in the overlap region are converted to non-linear region variables and used to evaluate the fluxes in the non-linear region. Likewise at every time step, all non-linear region variables are converted to linear acoustic region variables and used to evaluate the fluxes in the acoustic region. Freund et al. [10] pointed out that grid stretching and interpolation induce error in the calculation, though reducing the computing expense. The present simulation uses identical uniform grids in the overlapping region to avoid such generation of spurious waves.

\section{Results and Discussion}

The numerical simulation was conducted for a cold, nearly perfectly expanded axisymmetric supersonic jet of Mach number 2.1. The test case has been simulated previously [8] using large-scale equations. The total temperature of the jet is $294^{\circ} \mathrm{K}$, and the jet exit pressure is $0.0515 \mathrm{~atm}$. The Reynolds number based on exit conditions is approximately 70000. In the present calculation, the jet is excited at a Strouhal number of 0.2 with the Strouhal number defined as $S t=f D / U_{e}$, where $D$ is the nozzle exit diameter and $U_{c}$ is the jet exit centerline velocity.

The computational domain for this problem extends axially from $x / D=2.5$ to $x / D=35$, and radially from centerline to $r / D=16$, as shown in figure 1. Due to the steep mean flow gradient encountered at the jet exit, the computational grid was begun at an axial distance $x / D=2.5$ from the actual jet exit. The computational grid consists of 391 equally spaced points in the axial direction. In the radial direction, 150 points are used and stretched between centerline and $\mathrm{r} / \mathrm{D}=\mathbf{2 . 5}$ with concentration of grid points around $r / D=0.5$. Between $r / D=2.5$ and $r / D=16$, 130 equally spaced points are used with a spacing equal to that of the last stretched points. The interface between the non-linear and linear acoustic regions was chosen at $r / D=5$, above which the mean flow is uniform throughout the domain. 
Figure 2 shows the instantaneous distribution of $p$, $p, u$ and $v$ at $t=150$, where $t$ is the characteristic time defined as the ratio of nozzle exit radius to the jet exit centerline velocity. The horizontal line at $r / D=5$ in the figure indicates the boundary between the two domains. One can see smooth solutions across the matching region, although some binks are observed in the axial velocity distribution. The disturbances generated in the shear layer propagate through the matching region to the far field without any distortion. The wave-like nature of the flow field is evident and the solution is clean from boundary reflections.

Figure 3 shows contours of the root-mean-square values of the pressure and axial momentum distributions. The contour levels are 0.00035 and 0.0025 for the rootmean-square values of pressure and axial momentum respectively. No spurious disturbances are generated in the matching region, and the preferred forward emission is clearly shown in the figure.

Figures 4 shows the axial development of the axial momentum fluctuations on the nozzle lip line. The computed results obtained using the large-scale equations throughout the computational domain [8] are also shown in this figure. The present zonal approach gives the development of axial momentum fluctuations identical to those obtained in reference [8], as was expected. Figure 5 shows the axial development of the root-mean-square values of the pressure on the nozzle lip line. Again, the result of zonal approach is identical to those in reference [8].

Figure 6 compares the amplitude of pressure disturbance at $r / D=12$ along the axial direction at $S t=0.2$ with the results from reference [8]. Due to the use of linearized Euler equations in this region (no viscous dissipation), slightly higher peak was obtained by the present approach. The overall distribution is consistent with the previous large-scale computed results.

Figure 7 presents the pressure spectra at $x / D=25, \pi /$ $D=12$ for both zonal and large-scale approach. The amplitude at fundamental frequency, $S t=02$, is slightly higher for the zonal approach, while becomes lower at successive harmonics, than the large-scale simulation results. Previous linearized Euler computation along [29] did not show any generation of successive harmonics except for the fundamental one.

Figure 8 shows the sound pressure level distribution in the far field for the present and previous calculations. It is seen that the computed patterns of the sound pressure level contours are consistent with the previous work
[8]. As pointed out in reference [8], the computed results show a downstream shift of the lobes when compared to the experimental measurements. This can be explained that the calculations are for axisymmetric case, while the measurements showed that excited motion of the jet is dominated by the first helical mode in addition to the axisymmetric mode [24].

Figure 9 shows the calculated sound field directivity at a circle of radius $24 \mathrm{D}$ with center at the jet exit centerline. The angle is measured from the jet exit centerline. The calculated peak occurs around 15 degrees, which is the same as the one predicted by previous work [8].

The solution sensitivity to matching boundary location, which was chosen at $r / D=5$ and 7.5 respectively, is also investigated. Figure 10 shows the comparison of the axial distribution of root mean square values of pressure at $r / D=5$ and 7.5. As one can see that the solution is independent of the matching boundary location, which is denoted by $\mathrm{RM}$ in the figure.

\section{Conclusions}

A zonal approach for direct computation of sound generation and propagation from a supersonic jet is investigated. The computational domain is split into a nonlinear acoustic source generation regime and a linear acoustic wave propagation regime. The unsteady flow in the non-linear acoustic source region is governed by the large-scale equations, which are the filtered compressible Navier-Stokes equations. The linearized Euler equations are used to describe the sound wave propagation in the linear acoustic region. The computed results show that no spurious wave is generated in the matching region and the computational cost is reduced by $30 \%$ when compared with the direct simulation using large-scale equations alone. This substantial reduction in computational cost becomes significant when the approach is extended to three dimensional simulations.

\section{References}

1. Seiner, J. M., McLaughlin, D. K. and Liu, C. H., "Supersonic Jet Noise Generated by Large-Scale Instabilities," NASA TP-2072, September, 1982.

2. Zaman, K. B. M. Q. "Flow Field and Near and Far Sound Field of a Subsonic Jet," Journal of Sound and Vibration, Vol. 106, pp. 1-6, 1986.

3. Mankbadi, R. R. and Liu, J. T. C." "Sound Generated Aerodynamically Revisited: Large-Scale Structures in a Turbulent Jet as a Source of Sound," Philos. Trans. Royal Society of London A, Vol. 311, pp. 183-217, 1984. 
4. Mankbadi, R. R., "The Self Noise from Ordered Structures in a Low Mach Number Jet, "Journal of Applied Mechanics, Vol. 57, pp. 241-246, 1990.

5. Mankbadi, R. R., "Dynamics and Control of Coherent Structure in Tubulent Jets," Applied Mechanics Reviews, Vol. 45, No. 6, pp. 219-247, 1992.

6. Tam, C. K. W. "Jet Noise generated by Large-Scale Coherent Motion," Chapter 6 of "Aeroacoustics of Flight Vehicles: Theory and Practice. Vol. 1: Noise Source," edited by Hubbard, H. H., NASA RP-1258, 1991.

7. Mankbadi, R. R., Hayder, M. E and Povinelli, L. A. "The Structure of Supersonic Jet Flow and Its Radiated Sound," AIAA Journal, Vol. 32, pp. 897-906, 1994.

8. Mankbadi, R. R., Shih, S.H., Hixon, R. and Povinelli, L. A. "Direct Computation of Sound Radiation by Jet Flow Using Large-Scale Equations," AIAA Paper 95-0680, Reno, NV, Jan. 1995.

9. Shih, S. H., Hixon, R. and Mankbadi, R. R.,"Three Dimensional Structure in a Supersonic Jet: Behavior Near Centerline," AIAA Paper 95-0681, Reno, Nevada, January, 1995.

10. Iyrintzis, A. S. and Mankbadi, R. R., "On the Prediction of the Far-Field Jet Noise Using Kirchhoff's Formulation; AIAA Paper 95-0508, Reno, Nevada, January, 1995.

11. Freund, J. B., Lele, S. K. and Moin, P., "Matching of Near/Far-Field Equation Sets for Direct Computations of Aerodynamic Sound," AIAA Paper 934326, Long Beach, CA, Oct. 1993.

12. Smagorinsky, J., "General Circulation Experiments with the Primitive Equations, I. The Basic Experiment," Monthly Weather Review, Vol. 91, pp. 99164, 1963.

13. Edison, T. M., "Numerical Simulation of Turbulent Rayleigh-Bemard Problem Using Numerical Subgrid Modelling," Journal of Fluid Mechanics, Vol. 158, pp. 245-268, 1985.

14. Hardin, J. C., "Recent Insights into Computational Aero-Acoustics," in Computational Aero- and Hydro-Acoustics, edited by R. R. Mankbadi, A. S. Lyrintzis, O. Baysal, L. A. Povinelli, M. Y. Hussaini, FED-Vol. 147, pp. 1, 1993.

15. Gottlieb, D. and Turkel, E., "Dissipative Two-Four Methods for Time-Dependent Problems," Mathematics of Computation, Vol. 30, No. 136, pp. 703723, 1976.

16. Bayliss A. and Maestrello, L., "Simulation of Instabilities and Sound Radiation in a Jet," AIAA J., Vol. 19. pp. 835-841, 1981.

17. Maestrello, L. Bayliss, A. and Turkel, E., "On the Interaction of a Sound Pulse with the Shear Layer of an Axisymmetric Jet," J. of Sound and Vibration,
Vol. 74, pp. 281-301, 1981.

18. Frendi, A, Maestrello, L. and Bayliss, A, "Coupling Between a Supersonic Boundary Layer and a Flexible Surface," AIAA J., Vol. 31, pp. 708-713, 1993.

19. Frendi, A., Maestrello, L. and Bayliss, A., "Coupling Between Plate Vibration and Acoustic Radiation," J. of Sound and Vibration, Vol. 177, pp. 207226, 1994.

20. Ragab, S. A. and Sheen, S., "The Nonlinear Development of Supersonic Instability Waves in a Mixing Layer," Physics of Fluids A, Vol. 4, pp. 553-566, 1991.

21. Farouk, B., Oran, E. S. and Kailasanath, K., "Numerical Simulations of the Structure of Supersonic Shear Layers," Physics of Fluids A, Vol. 3, pp. 2786-2798, 1991.

22. Hixon, R., Shih, S. H., and Mankbadi, R. R., "Evaluation of Boundary Conditions for Computational Aeroacoustics." AlAA Paper 95-0160, Reno, Nevada, Jan. 1995.

23. Tam, C. K. W. and Burton, D.E., "Sound Generated by Instability Waves of Supersonic Flows, Part 2: Axisymmetric Jets," Joumal of Fluid Mechanics, Vol. 138, 1984, PP. 273-295, 1984.

24. Troutt, T. R. and McLaughlin, D. K., "Experiments on the Flow and Acoustic Properties of a Moderate Reynolds Number Supersonic Jet," Joumal of Fluid Mechanics, Vol. 116, PP 123-156, 1982.

25. Tam, C. K. W. and Webb, J. C., "Dispersion-Relation-Preserving Finite Difference Schemes for Computational Acoustics," Journal of Computational Physics, Vol. 107, pp. 262-281, 1993.

26. Bayliss, A. and Turkel, E., "Far Field Boundary Condition for Compressible Flows," Journal of Computational Physics, Vol. 48, pp. 182-199, 1982.

27. Enquist, B. and Majda, A., "Radiation Boundary Conditions for Acoustic and Elastic Wave Calculations," Communications on Pure and Applied Mathematics, Vol. 32, No. 3, pp. 313-357, 1979.

28. Hagstrom, L and Hariharan, S. I., "Far Field Expansion for Anisotropic Wave Equations," Computational Acoustics, Vol. 2, 1990.

29. Mankbadi, R. R., Hixon, R., Shih, S. H. and Povinelli, L. A., "On the Use of Linearized Euler Equations in the Prediction of Jet Noise," AIAA Paper 950505, Reno, Nevada, January, 1995. 


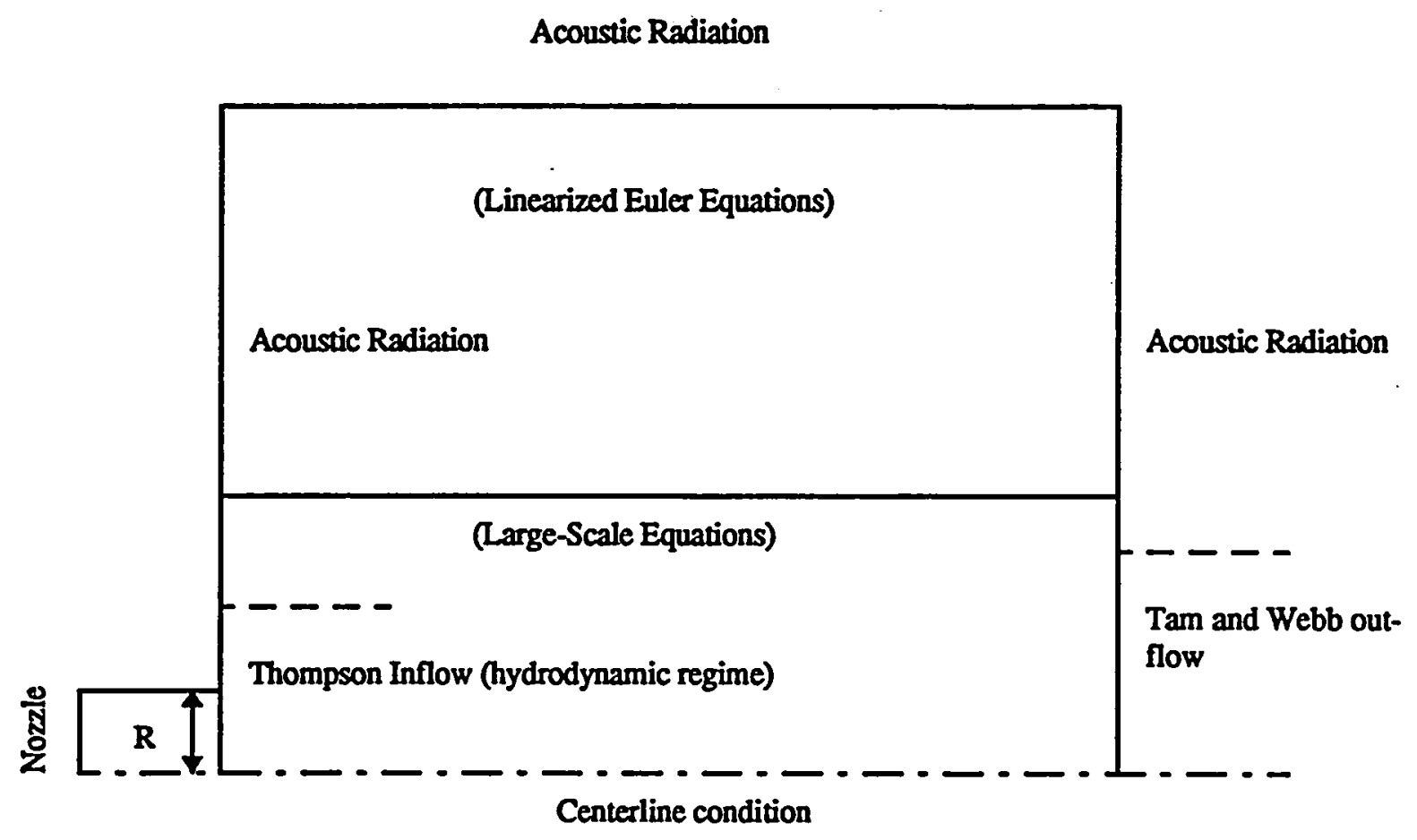

Fig. 1 Computational Domain 

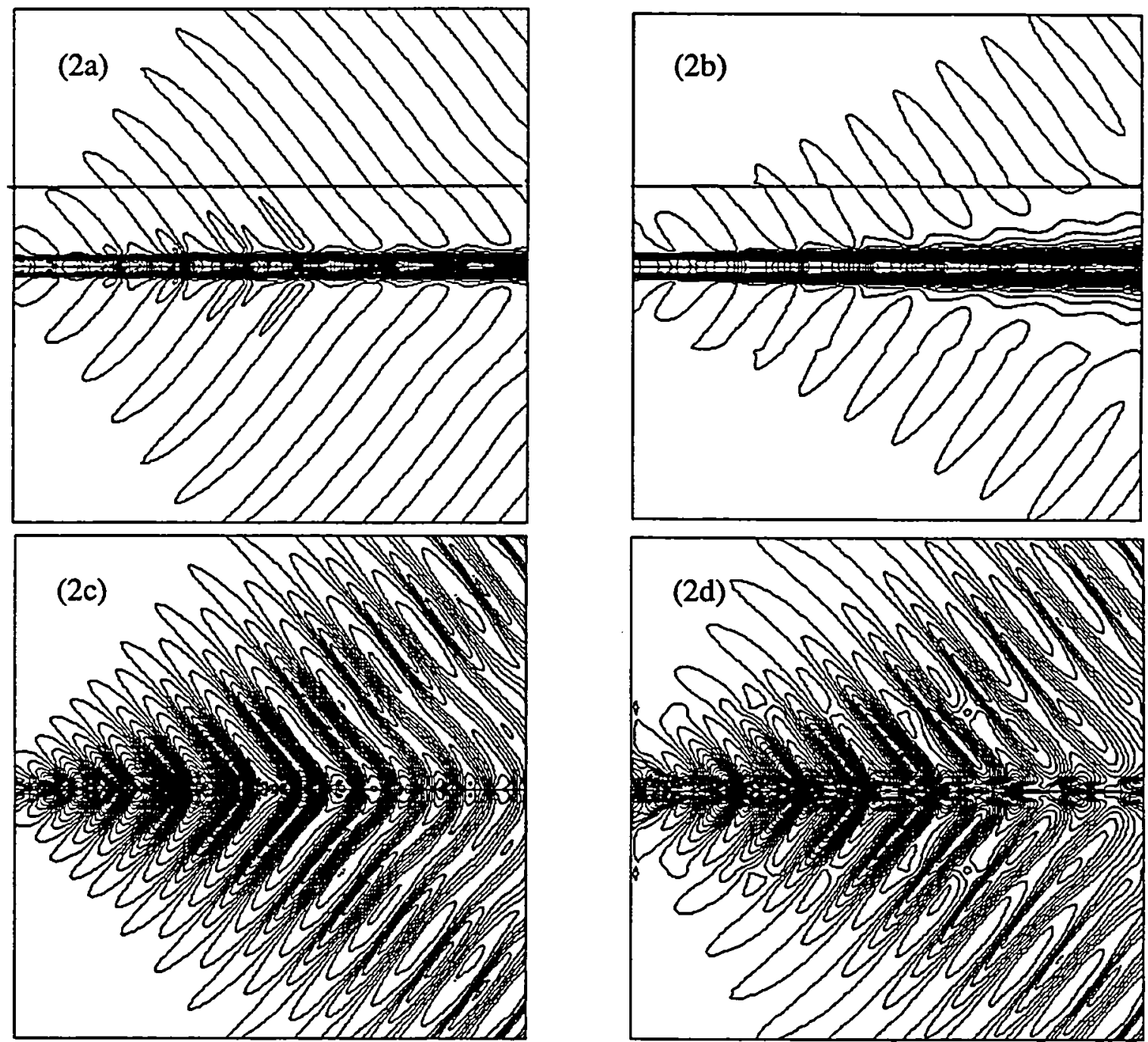

Fig. 2 Instantaneous distributions of (a) density (b) axial velocity (c) pressure (d) radial velocity, at $\mathrm{t}=150$.
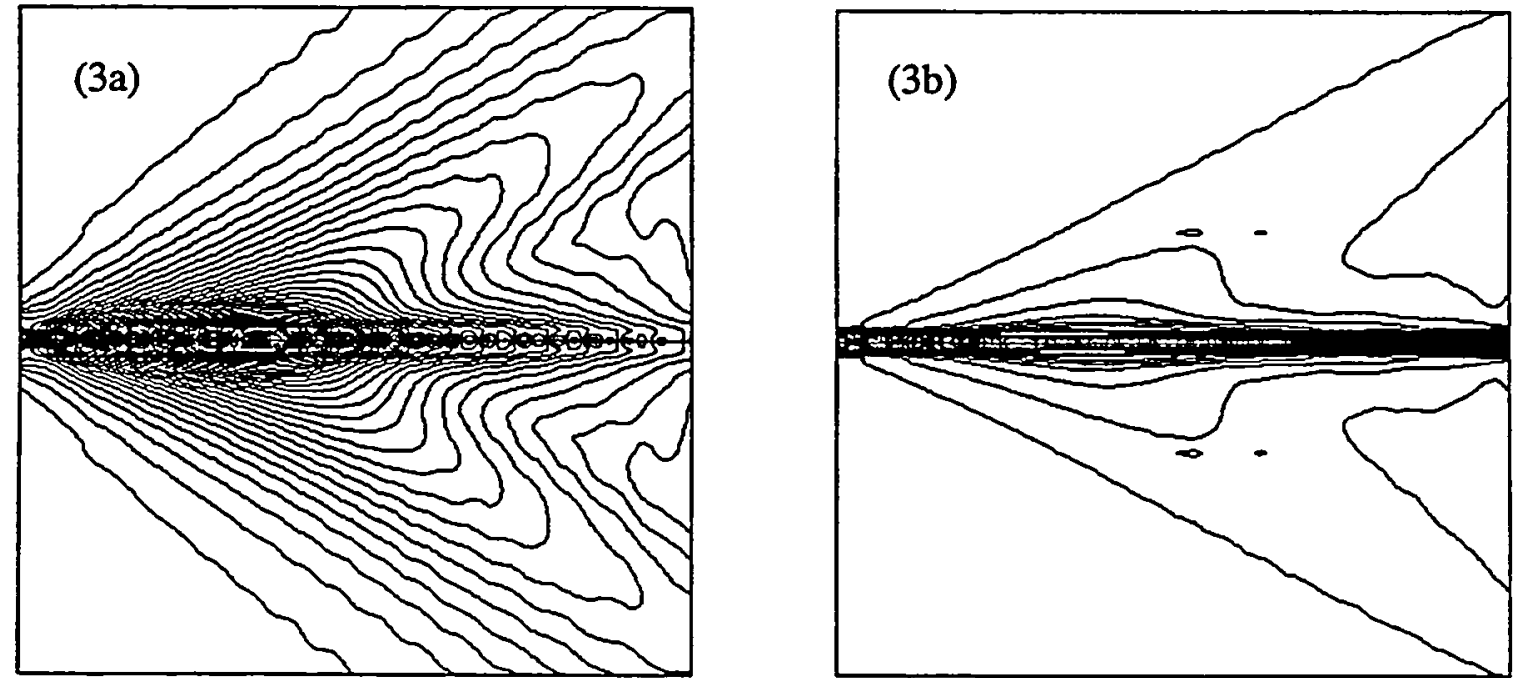

Fig. 3 Contours of root mean square values of (a) pressure (b) axial momentum. 


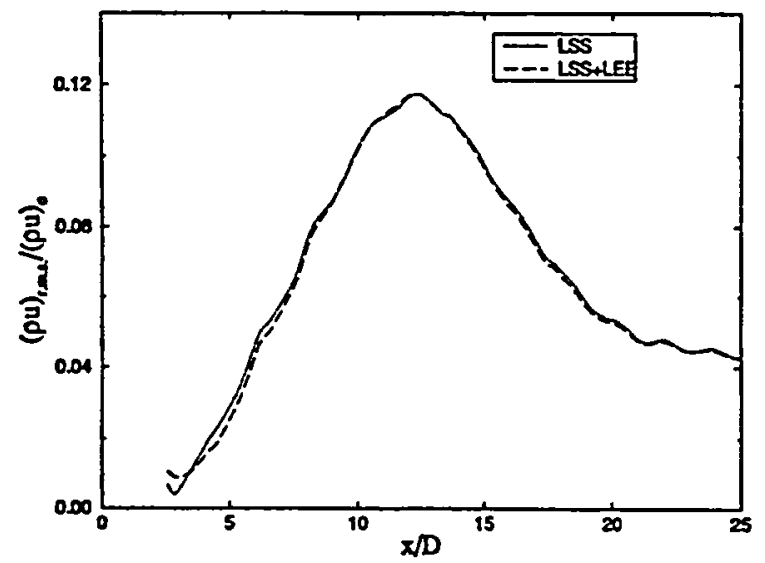

Fig. 4 Root mean square values of axial momentum at $\mathrm{r} / \mathrm{D}=0.5$.

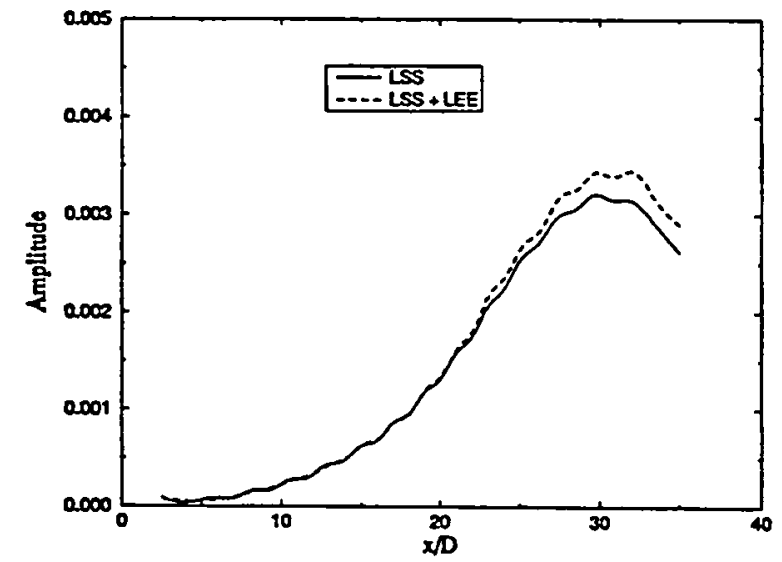

Fig. 6 Amplitude of pressure fluctuations at $r$ / $\mathrm{D}=12$.

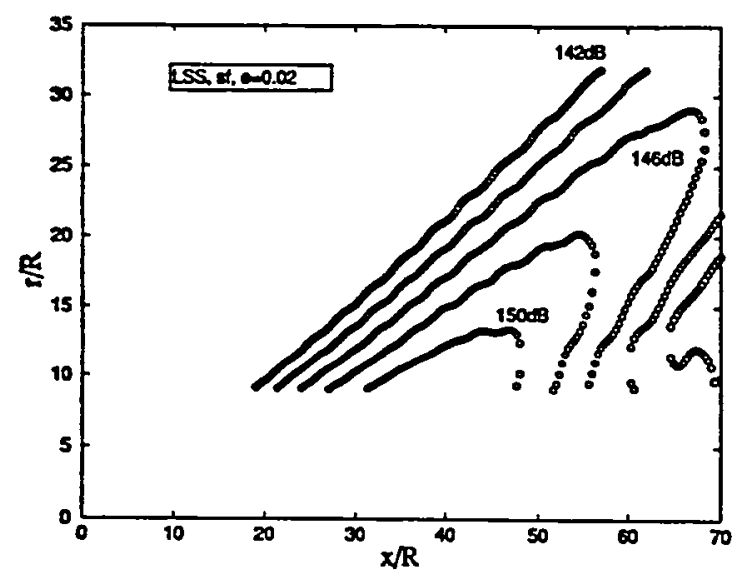

Fig. 8a Sound pressure level contours for large-scale calculations.

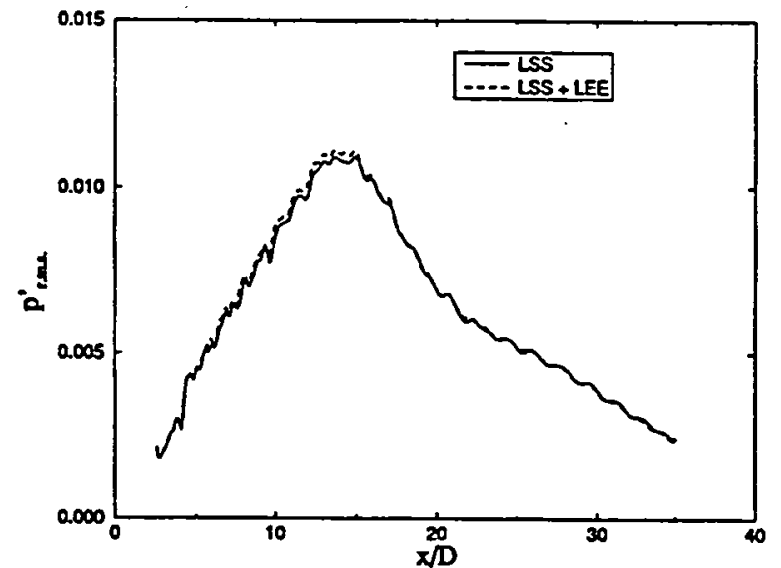

Fig. 5 Root mean square value of pressure at $\mathrm{r} / \mathrm{D}=0.5$.

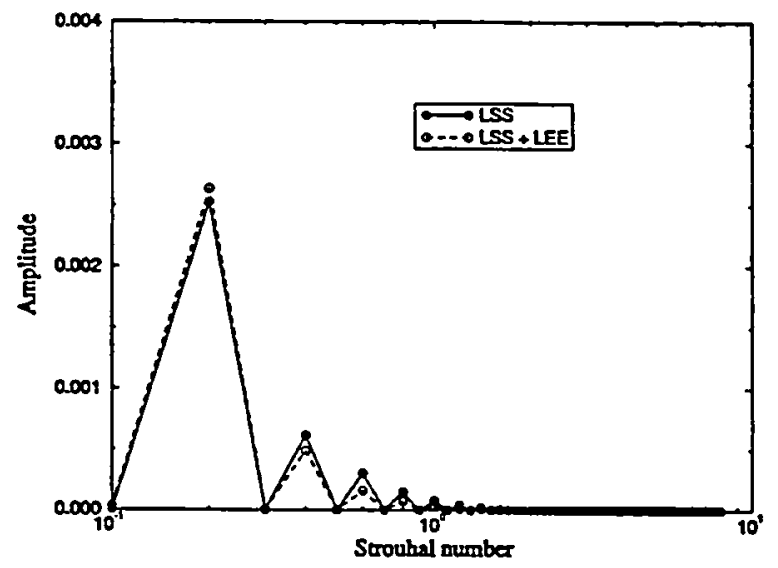

Fig. 7 Pressure spectra at $x / D=25, r / D=12$.

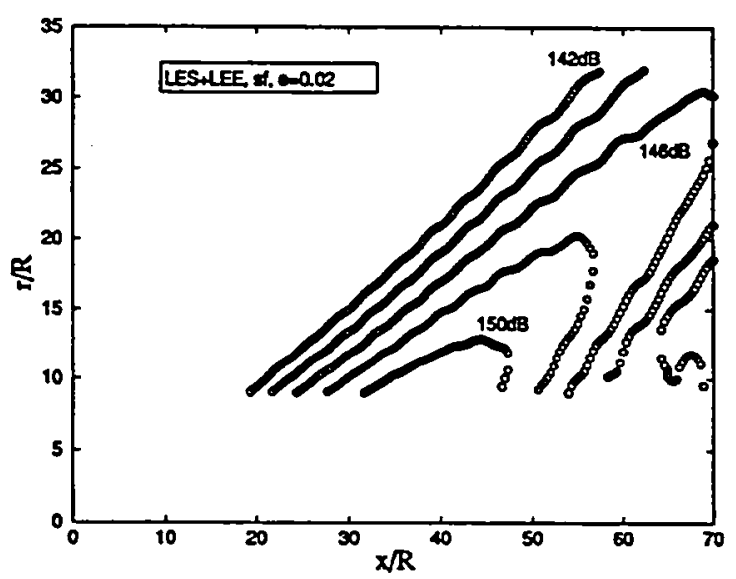

Fig. 8b Sound pressure level contours for largescale and linearized Euler calculations. 


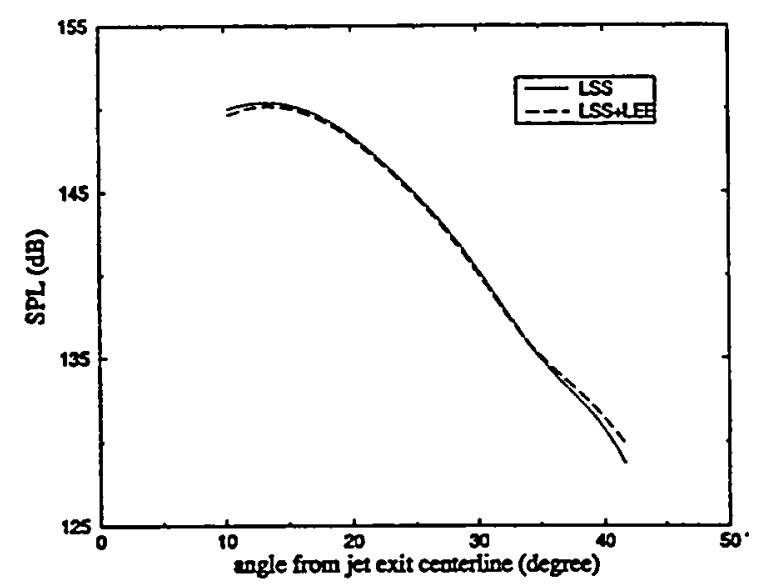

Fig. 9 Directivity of jet noise at $\bar{R} / D=24$.

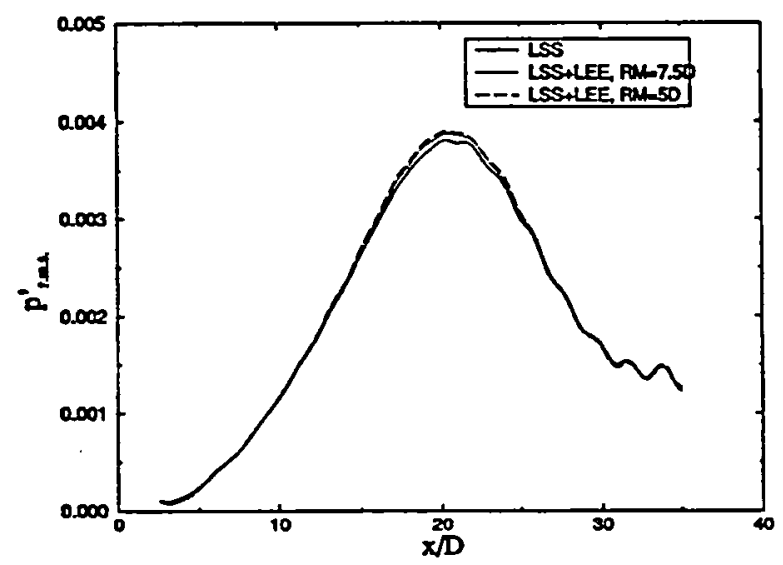

Fig. 10a Comparison of root mean square value of pressure at $\mathrm{r} / \mathrm{D}=5$.

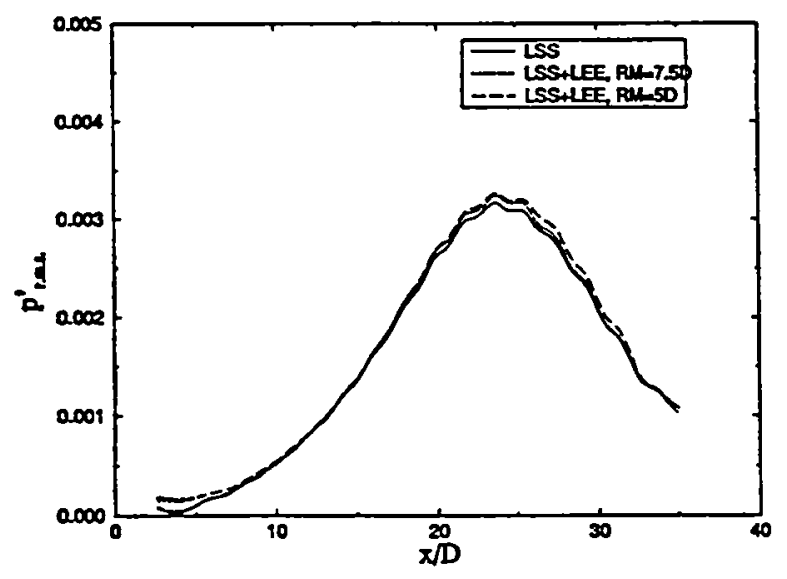

Fig. 10b Comparison of root mean square value of pressure at $\mathrm{r} / \mathrm{D}=7.5$. 
